\title{
T-ray spectroscopy of biomolecules
}

\author{
Bernd M. Fischer and Derek Abbott \\ School of Electrical \& Electronic Engineering and Centre for Biomedical Engineering \\ The University of Adelaide \\ Adelaide, SA 5005 \\ Email: bernd.fischer@adelaide.edu.au
}

\begin{abstract}
In the past few years it has been shown that the $\mathbf{T H z}$ spectra of many polycrystalline biomolecules show very distinct, characteristic features. The fact that these specific signatures can be used to identify many samples such as pharmaceuticals, illicit drugs, and explosives through a wide range of common packaging materials has spurred an increased commercial interest in THzTDS and imaging. However, most of these signatures that occur in the spectra of polycrystalline samples originate from phononlike vibrations of the well-ordered intermolecular structure. The spectra of larger biomolecules, due to the lack of such a long-range order, quite often show only a broad, featureless absorption. Furthermore, the sample preparation becomes more and more important, as many of these sample are only available in very small quantities or in liquid solutions. These hurdles constrict many biomedical applications of $\mathrm{THz}$ spectroscopy. In this presentation, we will show, based on a wide range of examples, the potential that THz-TDS and imaging hold for biomedical applications and discuss possibilities for overcoming the hurdles that currently obstruct their application.
\end{abstract}

\section{INTRODUCTION}

The characteristic signatures that dominate the $\mathrm{THz}$ (or $\mathrm{T}$ ray) spectra of most polycrystalline biomolecules are very sensitive to the molecular and crystalline structure. Therefore it is possible to differentiate and identify molecules of similar structure [1] and even their isomeric [2], [3] and crystalline configuration [4], [5]. This fingerprint ability and the low, featureless absorption of most typical packaging materials at $\mathrm{THz}$ frequencies are the basis for many biosensing applications. However, if the spectra lack such distinct signatures-as it is for example the case for amorphous samples [6], solutions, and most complex biomolecules even in the solid phase [7], [8]an identification of the sample based on recognition of sharp, specific spectroscopic features is often not possible. Yet, a differentiation between for example a test sample and a known reference sample is still feasible, and for many biomedical applications such differentiations are sufficient.

\section{EXPERIMENTAL}

The spectra that will be shown in this presentation have been recorded using a custom built THz-TDS spectrometer in transmission and reflection mode (see for example [9]) and a commercially available fibre coupled system (Picometrix Tray 2000) that can be used either in transmission or reflection mode.

\section{RESULTS}

In order to demonstrate the high sensitivity of $\mathrm{THz}$ spectra to small differences in the molecular structure we show

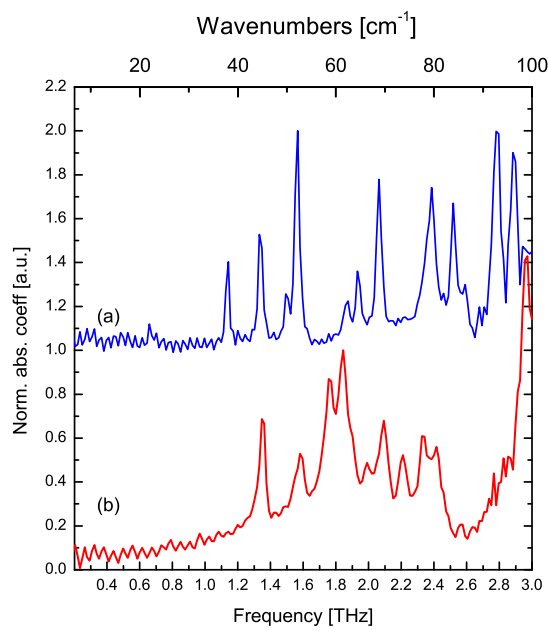

Fig. 1. Normalized Absorption of polycrystalline (a) estrone and (b) estriol (shifted vertically for better visibility). Both spectra show several sharp, distinct spectral features. These features differ clearly for both samples, despite the pronounced similarity in the molecular structure. These characteristic signatures can then be used to identify the sample even through many typical unpolar packaging materials.

in Figure 1 the $\mathrm{THz}$ spectra of two hormones, estrone and estriol, recorded at $10 \mathrm{~K}$. Despite the pronounced similarity of the molecular structure, the spectra differ significantly from each other. As most of the the low-frequency vibrations that give rise to the characteristic features in the $\mathrm{THz}$ range are of intermolecular rather than intramolecular character, THzTDS can also be used to distinguish and identify different crystalline configurations [5]. This ability of THz-TDS for chemical recognition [7] has spurred the increased interest in THz-TDS for applications such as pharmaceutical quality control and detection of explosives and illicit drugs.

However, the spectra of most amorphous samples, liquids [10] and solutions, and also of many complex biomolecules in the solid state [8], [11] lack such pronounced characteristic features. Therefore, spectral identification based on the recognition of the characteristic spectral fingerprints is often impossible for such samples. As example, we show in Figure 2 a typical absorption spectrum of a polypeptide, recorded at ambient temperature. Compared for example to the richly structured spectra of the polycrystalline hormone 


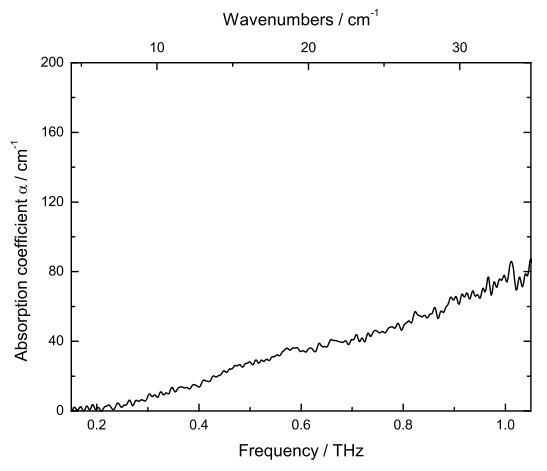

Fig. 2. Absorption spectrum of a polypeptide. Compared to the richly structured spectra of most polycrystalline samples, only a broad, featureless slope is observed.

samples shown in Figure 1 only a broad, featureless absorption is observed. Yet, by applying a careful sample preparation in order to avoid any artifacts arising from uncertainties in thickness, water content, density, and changes in the conformational state induced by inappropriate sample handling, the slope and absolute value of the absorption coefficient can still be used to differentiate between different samples such as for example a test substance and a reference sample. For example, we have recently shown that it is possible to differentiate between two different artificial RNA strands, Poly-A and Poly-C [11]. In this presentation, we will discus the potential of THz-TDS for detecting structural differences in complex biomoecules such as for example polypetides. By applying careful sample preparation, increasing the SNR [12] and applying advanced signal processing techniques [13], we can demonstrate that despite the lack of distinct signatures in the spectra of complex biomolecules $\mathrm{THz}$ spectrosopy and imaging are still versatile tools for biosensing and biomedical applications.

\section{CONCLUSION}

The THz spectra of small, polycrystalline biomolecules show very characteristic features. These $\mathrm{THz}$ signatures are the basis for many recent applications of T-ray spectroscopy and imaging. For larger molecules, due to the lack of such characteristic signatures, these fingerprinting techniques are in many cases not applicable. However, a comparison of the absolute value and slope of the absorption spectra can still lead to valuable information about structural and conformational properties of the samples.

\section{ACKNOWLEDGMENT}

The authors would like to thank Hanspeter Helm and Sam P. Mickan for fruitful discussions. We would further like to thank the BMBF, the Australian Research Council, and the Sir Ross and Sir Keith Smith foundation for financial support.

\section{REFERENCES}

[1] B. Fischer, M. Walther, and P. U. Jepsen, "Far-infrared vibrational modes of DNA components studied by terahertz time-domain spectroscopy," Phys. Med.Biol., vol. 47, pp. 3807-3814, 2002.
[2] M. Walther, B. Fischer, M. Schall, H. Helm, and P. U. Jepsen, "Farinfrared vibrational spectra of all-trans, 9-cis and 13-cis retinal measured by THz time-domain spectroscopy," Chem. Phys. Lett., vol. 332, pp. 289-295, 2000.

[3] P. C. Uphadya, Y. C. Shen, A. G. Davies, and E. H. Linfield, "FarInfrared vibrational modes of polycrystalline saccharides," Vibrational Spectroscopy, vol. 35, pp. 139-143, 2004.

[4] P. F. Taday, I. V. Bradley, D. D. Arnone, and M. Pepper, "Using terahertz pulse spectroscopy to study the crystalline structure of a drug: a case study of the polymorphs of rantidine hydrochloride," J. Pharm. Sci, vol. 92 , pp. $831-838,2003$.

[5] B. M. Fischer, M. Franz, and D. Abbott, "T-ray biosensing: a versatile tool for studying low-frequency intermoecular vibrations," in Proceedings of SPIE: Biomedical Applications of Micro- and Nanoengineering III, vol. 6416, art. no. 64160U, 2006.

[6] M. Walther, B. M. Fischer, and P. U. Jepsen, "Noncovalent intermolecular forces in polycrystalline and amorphous saccharides in the far infrared," Chemical Physics, vol. 288, pp. 261-268, 2003.

[7] B. Fischer, M. Hoffmann, H. Helm, G. Modjesch, and P. U. Jepsen, "Chemical recognition in terahertz time-domain spectroscopy and imaging," Semicond. Sci. Technol., vol. 20, pp. 246-253, 2005.

[8] J. Knab, J. Y. Chen, and A. G. Markelz, "Hydration dependence of confirmational dielectric relaxation of lysozyme," Biophysical Journal, vol. 90, no. 7, pp. 2576-2581, 2006.

[9] P. U. Jepsen and B. M. Fischer, "Dynamic range in terahertz timedomain transmission and reflection spectroscopy," Optics Letters, vol. 30, no. 1, pp. 29-31, 2005.

[10] S. Schrödle, B. Fischer, H. Helm, and R. Buchner, "Picosecond dynamics and microheterogenity of water + dioxane mixtures," The Journal of Physical Chemistry A, vol. 111, pp. 2043-2046, 2007.

[11] B. M. Fischer, M. Hoffmann, H. Helm, R. Wilk, F. Rutz, T. KleineOstmann, M. Koch, and P. U. Jepsen, "Terahertz time-domain spectroscopy and imaging of artificial RNA," Optics Express, vol. 13, no. 14, pp. 5205-5215, 2005.

[12] J. Belakrishnam, B. M. Fischer, S. P. Mickan, and D. Abbott, "Investigation on imroving the noise performance of t-ray liquid spectroscopy via double-modulated differential time-domain spectroscoy," in Proceedings of SPIE: Biomedical Applications of Micro and Nanoenineering, vol. 6416, art. no. 6410V, 2007.

[13] X. Yin, B. W.-H. Ng, B. Fischer, B. Ferguson, S. P. Mickan, and D. Abbott, "Feature extraction from terahertz pulses for classification of RNA data via support vector machines," in Proceedings of SPIE: Microand Nanotechnology: Materials, Processes, Packagin and Systems III, vol. 6415 , art. no. 641516, 2006. 